\title{
Building an Engineering-Based Medical College: Is the Timing Ripe for the Picking?
}

\author{
Lawrence S. Chan ${ }^{1}$
}

Published online: 7 December 2015

(C) International Association of Medical Science Educators 2015

\section{Background}

The current US healthcare faces two major challenges in that while the cost is very high its overall outcome is not excellent. In its 2014 report, the Organization for Economic Cooperation and Development (OECD) informed us that healthcare expenditure of the USA costs $16.9 \%$ gross domestic products (GDP) in 2012, the highest of all OECD countries [1]. Yet according to a 2014 study conducted by the Commonwealth Fund, the USA is ranked the very last in overall healthcare quality among 11 nations including Australia, Canada, France, Germany, the Netherlands, New Zealand, Norway, Sweden, Switzerland, the United Kingdom, and the USA [2]. Specifically, the USA is last or near last on measures of access, efficiency, and equity [2]. Furthermore, the current medical practice is outpaced by the rapid advancement of health science and technology. A recent survey on genetic curricula in USA and Canadian medical schools found that only $26 \%$ responders reported formal genetic teaching during third and fourth year of school, and most responders felt the amount of time spent on genetics was insufficient for future clinical practice in this era of genomic medicine [3]. Not only medical educators felt the current medical education lags, students at Harvard Medical School expressed similar sentiment [4]. They felt that "our ability and capacity to train both new and experienced clinicians to manage the tremendous amount of data lag far behind the pace

Lawrence S. Chan

larrycha@uic.edu

1 Department of Dermatology, University of Illinois College of Medicine, 808 South Wood Street, R380, MC624,

Chicago, IL 60612, USA of the data revolution" and that "medical education at all levels must come to address data management and utilization issues as we enter the era of Big Data in the clinical domain" [4]. In addition, recent studies have pointed out that advanced technology useful to teach undergraduate medical knowledge and skills such as sonography and otolaryngology was underutilized $[5,6]$. Since undergraduate medical education is the exclusive physician pipeline and the gateway to the future of medicine, what we educate the medical students today would have a profound influence what the physicians will practice in the 50 years ahead. The most effective way to transform medicine of the future is, therefore, through medical education reform. Comparing the dynamic development of health science and technology and relative static medical education, one wonders if today's medical education would be optimal in nurturing future physicians. Furthermore, the combined findings of low efficiency and high expenditure of the US health system has prompted the Institute of Medicine (IOM) to call for the implementation of three aims of medicine in the future: "better care, better health, and lower costs" [7, 8]. In his commencement speech delivered at the University of Illinois College of Medicine on May 8th, 2015, Dr. Victor Dzau, President of IOM, encouraged the medical graduates to "be innovative, challenge the status quo, think out of the box, and make a difference!" The news of first Engineeringbased medical school, Carle-University of Illinois College of Medicine, to be established at the University of Illinois Urbana/Champagne Campus has triggered various reactions within academic medicine community [9]. An engineeringbased medical education curriculum, which intends to promote innovation, creativity, and efficiency, could indeed be a roadmap to the future of medicine. In this commentary article, the goal of engineering education will be defined, followed by an attempt to solidify the meaning of engineering-based medical education, and then the rationale for the engineering- 
based medical education will be discussed. Although a growing physician go through two stages of training, the medical school education followed by a post-graduate residency, this article focuses on the former.

\section{Discussion}

\section{Engineering Education Defined}

As the first step to debate the issue of "engineering-focused medical education," it will be prudent that we first define what "engineering education" is consisted of. Mentioning the word "engineering" would naturally evoke an image of a bridge (civil engineering), an automobile (mechanical engineering), a chemical plan (chemical engineering), or a computer (electrical engineering). While engineering is indeed rooted in the foundation of and is synonymous with problem solving and technology, the technique of apply scientific discovery effectively and efficiently to human use, the actual goal of engineering education is much broader.

The goal of engineering education is delineated in the documents of the American Society of Engineering Education (ASEE), the most important intellectual think tank and the policy-shaping body of engineering education in the USA. In the most recent document entitled "Transforming undergraduate education in engineering," the ASEE determined 15 key areas of "Knowledge (K), Skills (S), and Abilities (A)" as the priorities for reforming the future engineering education [10] (Table 1).

\section{Engineering-Based Medical Education Defined}

Having defined "engineering education," let us now attempt to define "engineering-based medical education." Although a scholarly definition of an engineering-focused medical education is currently not available, we may be able to characterize it by examining the mission statements of education institutes with combined goals of engineering and medicine.

Established in 1995 at the Massachusetts General Hospital, the Center for Engineering in Medicine declared "The mission of the Center for Engineering in Medicine is twofold: first, to train MDs, PhDs, and predoctoral students in the fundamentals of biomedical engineering; and second, to bring the principles and tools of biomedical engineering to the forefront of biomedical research and patient care."

The Engineering in Medicine Program coordinated by the Thayer School of Engineering and the Geisel School of Medicine at Dartmouth University offering both combined $\mathrm{MD} / \mathrm{MS}$ and $\mathrm{MD} / \mathrm{PhD}$ programs in medicine and bioengineering describes its educational goal: "Engineering in medicine research addresses today's technology-driven healthcare system. Advances depend not only on clinical expertise but also on those trained to look at the technical side of patient care."

Created in 1996, the Institute for Medicine and Engineering at the University of Pennsylvania provides this mission statement: "The mission of the Institute for Medicine and Engineering is to stimulate fundamental research at the interface between biomedicine and engineering/physical/computational sciences leading to innovative applications in biomedical research and clinical practice."

Similarly, the Biomedical Engineering Department at Yale School of Engineering and Applied Science posted this educational goal: "Biomedical Engineering at Yale has two related goals: first, the use of the tools and methods of engineering to better understand human physiology and disease; second, the development of new technologies for diagnosis, treatment, and prevention of disease."

With the above statements examined, an engineering-based medical education may be characterized as an educational system that aims to teach and train medical students not only the traditional theory and practice of patient care but also the principles of biomedical engineering in innovative and efficient approaches to health care delivery and in state-of-the-art technology utilization for understanding, diagnosis, treatment, and prevention of human diseases.

Another way to characterize engineering-based medical education is to view the two educational goals depicted by the national first engineering-based medical college, CarleUI College of Medicine. These two goals are (1) re-invent health care around revolutionary advances in engineering and technology to further research, education, and health care delivery; and (2) transform health care education of physicians through the development of team-based innovative approaches to achieving improved health care outcomes through the continuum of care: preventive medicine, chronic disease management, acute care, rehabilitative medicine, and end-oflife care [11]. Instead of the traditional discipline-based approach (like anatomy, biochemistry, etc.), this curriculum would converge medicine and engineering, quantitative and computer sciences, and technology to teach human body as an integrated system that is critical to the development of analytical and problem-solving skills. Engineering technologies and approaches would be incorporated throughout the entire 4year curriculum. For example, in the course of microbiology, its curriculum will teach an understanding of microbes and engineering approaches to the alteration of genomes and biological circuits. In the computer science course, its curriculum will educate students the data mining methods and patient care guideline development. And in the clinical training, its curriculum will incorporate 3D printing, bioreactor, and advanced analysis techniques [11]. To provide an illustration of a general framework in an engineering-based medical education curriculum, Table 2 depicted a proposed Year I Curriculum 
Table 1 The goals of education reform in engineering dovetail with the goals of education reform in medicine

\begin{tabular}{|c|c|c|c|}
\hline Engineering education reform ${ }^{\mathrm{a}}$ & Item no. & Medicine education reform ${ }^{\mathrm{b}}$ & Item no. \\
\hline Good communication skills (S) & 1 & $\begin{array}{l}\text { Rigid time frame in school to flexible competency-based } \\
\text { training }\end{array}$ & 1 \\
\hline Physical sciences and engineering science fundamentals $(\mathrm{K})$ & 2 & Individual physicians to interprofessional teams & 2 \\
\hline Skills to identify, formulate, and solve engineering problems (S) & 3 & $\begin{array}{l}\text { Low technology utilization to high technology } \\
\text { utilization }\end{array}$ & 3 \\
\hline Systems integration $(\mathrm{K})$ & 4 & Inpatient focus to outpatient focus & 4 \\
\hline Curiosity and persistent desire for continuous learning (A) & 5 & Individual patient advocacy to population health outcome & 5 \\
\hline Self-drive and motivation (A) & 6 & Limited focus on cost to stewardship of resources & 6 \\
\hline Culture awareness in the broad sense $(\mathrm{K})$ & 7 & Understanding role in health care delivery & 7 \\
\hline Economics and business acumen $(\mathrm{K})$ & 8 & & \\
\hline $\begin{array}{l}\text { High ethical standards, integrity, and global, social, intellectual, and } \\
\text { technological responsibility (A) }\end{array}$ & 9 & & \\
\hline Critical thinking (S) & 10 & & \\
\hline Willingness to take calculated risk (A) & 11 & & \\
\hline Efficiency for prioritization (S) & 12 & & \\
\hline Project management $(\mathrm{S})$ & 13 & & \\
\hline Teamwork skills and function on multidisciplinary teams (A) & 14 & & \\
\hline Entrepreneurship and intrapreneurship (A) & 15 & & \\
\hline
\end{tabular}

${ }^{a}$ Goals established by the American Society of Engineering Education (ASEE)

${ }^{\mathrm{b}}$ Goals set by the American Medical Association (AMA)

Table 2 Year I proposed curriculum in an engineering-based College of Medicine (Carle-UI)

Proposed course contents

\section{Fall term courses}

Mathematical, statistical, and modeling methods for medicine

Visualization of human functional anatomy

Human system pathology

Biochemistry and biotechnology for metabolic diseases

Molecular biology and genomics

Immunology for molecular medicine

Team-based problem-based learning

January term courses

Musculoskeletal biomechanics and pathophysiology

Molecular biomarkers in health and disease

Evidence-based informatics for medicine

Technology for patient care

Medical research methods

Spring term courses

Systems and network in endocrinology

Nanotechnology in hematology

Biomechanics: cardiovascular pathophysiology

Biomechanics: respiratory pathophysiology

Biomechanics: renal pathophysiology

Medical decision making for patient care

Personalized, mobile, and global medicine

Team-based problem-based learning
Math; stats, probability, computer programming, simulations Advanced visualization tech; imaging; simulations Systems-level approaches, advanced microscopy Biotechnology assays, laboratory testing Genomics; bioinformatics; BIG DATA analysis Molecular targeting for drugs and imaging Modules to introduce clinical problem early

Biomechanics (muscle, cellular); tissue engineering Biomarkers - molecular, imaging, signals, symptoms, signs Review, analyze, assimilate, and use the literature Electronic medical record; mobile health diagnostics Methods of research; IRBs; ethics; professionalism

System biology; networks; feedback; control; analysis Nanotechnology; nanomedicine; bioMEMS

Quantitative modeling; simulations; fluid mechanics Quantitative modeling; simulations; dynamics

Biomaterials; electrolytes; natural/synthetic interface medical decision analysis from clinical/diagnostic data Low-cost technology; public/global education and health Modules introducing clinical problems early

IRBS internal review board, bioMEMS biological microelectromechanical system 
of the MD program at Carle-University of Illinois College of Medicine [11].

\section{Rationale for Engineering-Based Medical Education}

Why then would we need an engineering-based medical education? Although some teaching method changes have been made through Liaison Committee on Medical Education mandates recently, medical education by and large has been in a static state, lacking behind the rapid changes in science, technology, and society. Some issues include:

- Medical students are largely taught to be an individual patient advocate, rather than thinking critically about being steward of the entire health care system, particularly in terms of resources. This issue has prompted the IOM to call for lowering health care cost [8]. The engineeringbased medical education could provide solution by educating students the efficient methods in healthcare delivery.

- Medical students are primarily taught in providing care: in making correct diagnosis and in prescribing right medication or surgical procedure, and not much about other determinants that are also significant for improving health. For example, population studies estimated that medical care contribute only about $10 \%$ of the variance in the final outcome of health, whereas a huge percentage $(50 \%)$ of the outcome is dependent on behavior and social factors [12]. Yet physicians are playing little role in the other factors. This issue has prompted the Association of American Medical Colleges to call for the inclusion of public health in medical education curriculum [13]. In fact, the University of Wisconsin School of Medicine has changed its name to "University of Wisconsin School of Medicine and Public Health" in 2005 to involve students in health promotion and disease prevention. The engineering-based medical education, with its emphasis on global perspective, could promote holistic approach and resource stewardship in our future physicians.

- Medical students are predominantly taught to be excellent individual physicians, rather than being a team member of a large health care system. This issue has prompted the American Medical Association (AMA) to call for the promotion of teamwork concept [14]. With its educational training of teamwork, the engineering-based medical curriculum could instill the teamwork concept into the daily practice of our future physicians.

- Rapid advancement of biomedical technology has occurred at a much faster pace than the current medicine practice $[15,16]$. Specifically, the participants of Translatel 2014 meeting in Berlin Germany have reached a consensus on the rate-limiting factor for advancing translational medicine and made an urgent statement: "The pace of basic discoveries in all areas of biomedicine is accelerating. Yet translation of this knowledge into concrete improvements in clinical medicine continues to lag behind the pace of discovery" [15]. Three recent biomedical articles published in 2015, randomly chosen as below, demonstrate the contribution of bioengineering to medicine and illustrate the current state of medical science advancements that are not universally taught in our medical schools:

$3 \mathrm{D}$ printing technology is used in the treatment of structure malformation. The biomedical engineers at the University of Michigan have successfully implanted customized patient-specific 3D-printed external airway splints in three infants suffered from tracheobronchomalacia, a lifethreatening condition of excessive collapse of airways during respiration. As a result, this 3D-printed material eliminated the airway disease, strongly illustrating the biotechnology contribution to medicine $[17,18]$.

Nanotechnology is utilized in gene editing to correct genetic mutation. The Yale University scientists have derived a gene editing technique, which a synthetic molecule similar to DNA, called peptide nucleic acids (PNAs), and together with donor DNA are utilized to correct a genetic defect in cystic fibrosis. Using biodegradable microscopic nanoparticles to facilitate the delivery of PNA/ DNA to the target cells, the Yale researchers were able to trigger the DNA repair and recombination pathway in these target cells, resulting the proper gene correction in both human airway cells and mouse nasal cells, as well as in nasal and lung tissues, significantly demonstrating the nanotechnology contribution to medicine $[19,20]$. Mobile phone video microscope is applied in parasitic infection detection. Researchers at the University of California at Berkeley and NIH have together produced a mobile phone automatic microscopic device which can be used by health providers in low-resource areas of Africa to detect filarial parasite Loa loa, using whole blood loaded directly into a small glass capillary from a simple finger stick, without the need for conventional sample preparation or staining. This device can now be used to exclude patients infected with $L$. loa from lifethreatening medication side effects of ivermectin in just $2 \mathrm{~min}$, while the remaining mass population can be safely administered with ivermectin to eliminate the debilitating onchocerciasis and lymphatic filariasis [21].

The AMA is keenly aware of these educational deficiencies and promotes medical education reform in an article entitled "Accelerating change in medical education" on its website [14]. The AMA urged seven changes of present-day medical practice in order to adapt to the future health care, through medical education reform [14] (Table 1). 
Comparing these 7 changes urged by the AMA and the 15 future engineering education priorities proposed by the ASEE, one finds amazingly parallel goals between these two educational organizations [10, 14]. Specifically, items 2, 5, 6, and 7 of educational changes supported by the AMA correspond to items 14 (team work), 9 (global perspective), 9 (stewardship), and 4 (system practice) of the educational reform priorities proposed by the ASEE, respectively. Item 3 of the change supported by the AMA is the core component of engineering education (high use of technology). Such similarity of educational goals between engineering and medicine should relieve fear that educating medical students through an engineeringbased curriculum may lead future physicians into the wrong path of impersonal technicians [22]. Nevertheless, due to the increase curriculum load in technology, an engineering-based medical education would be better served by including courses of humanity. For example, a first year course termed "Profession of Medicine" taught at the Geisel School of Medicine at Dartmouth University introduces its students to some of the complex human issues in medicine, such as disability and end-of-life care with real patient encounter, aiming to teach students to deal with patients compassionately [23]. A Medical Arts program established in 2009 at the University of Michigan School of Medicine, which introduces the students to the humanities and arts, might also be a good model [24].

Furthermore, in light of the call for "better care, better health, and lower costs" of the US health system by the IOM, one would appreciate the value of adding the engineering's efficiency-oriented approach of problem solving to the curriculum of medical education $[8,10]$. Another innovative program that can be incorporated into the engineering-based medical college curriculum will be a field-tested medical technology design project [25]. In this 6-month-long program, medical students were partnered with business, law, design, and engineering students to form interdisciplinary teams aiming to develop practical solution for unmet medical needs and were provided with prototyping fund, access to clinical and industry mentors, development facilities, and didactic teachings in ideation, design, intellectual property, FDA regulation, prototyping, market analysis, business planning, and capital acquisition. After a period of 4 years, the 396 participants have developed 91 novel medical devices, and helped launching 24 new companies [25]. This kind of project would likely teach our future physicians lessons on teamwork, innovation, and solution-oriented medical practice.

\section{Summary}

Therefore, since an engineering-based medical education would dovetail the future goals and objectives of medical education in such substantial way, it could be considered as a good pathway for transforming our future medical education. Collectively, an engineering-based medical education would fulfill the medical education reforms called for by the IOM, AMA, and AAMC. The timing may be ripe now to test this concept in a small number of institutions.

Acknowledgments This academic work is supported by Dr. Orville J. Stone Professorship (L.S.C., University of Illinois).

\section{References}

1. OECD health statistics 2014. How does the United States compare? www.oecd.org/unitedstates. Accessed 13 September 2015.

2. Mirror, mirror on the wall, 2014 update: how the U.S. health care system compares internationally. www.commonweathfund.org/ publications. Accessed 13 September 2015.

3. Plunkett-Rondeau J, Hyland K, Dasgupta S. Training future physicians in the era of genomic medicine: trends in undergraduate medical genetic education. Genet Med. 2015. doi:10.1038/ gim.2014.208.

4. Moskowitz A, McSparron J, Stone DJ, Celi LA. Prepare a new generation of clinicians for the era of Big Data. Harvard Medical Student Review. 2015 January 3. www.hmsreview.org. Accessed 24 August 2015.

5. Day J, Davis J, Riesenberg LA, et al. Integrating sonography training into undergraduate medical education: a study of the previous exposure of one institution's incoming residents. J Ultrasound Med. 2015;34:1253-7.

6. Fung K. Otolaryngology-head and neck surgery in undergraduate medical education: advances and innovations. Laryngoscope. 2015;125 Suppl 2:S1-14.

7. Munro D. U.S. healthcare ranked dead last compared to 10 other countries. Forbes. June 16, 2014. www.forbes.com. Accessed 27 June 2015

8. Core measurement needs for better care, better health, and lower costs: counting what counts-workshop summary. Institute of Medicine. June 24, 2013. www.iom.edu. Accessed 25 June 2015.

9. Cohen JS. U. of I. trustees approve new medical school. Chicago Tribune. March 12, 2015. www.chicagotribune.com. Accessed 25 June 2015.

10. Transforming undergraduate education in engineering. American Society for Engineering Education. 2014. www.asee.org. Accessed 25 June 2015.

11. New College. New Medicine. The first college of medicine specifically designed at the intersection of engineering and medicine. https://medicine.illinois.edu/news.html. Accessed 17 September 2015.

12. Hershberger PJ, Bricker DA. Who determines physician effectiveness? JAMA. 2014;312:2613-4.

13. Public health in medical education online community of practice. Association of American Medical Colleges. www.aamc.org. Accessed 25 June 2015.

14. Accelerating change in medical education. American Medical Association. www.ama-assn.org. Accessed 25 June 2015.

15. Duda GN, Grainger DW, Frisk ML. Changing the mindset in life sciences toward translation: a consensus. Sci Transl Med. 2014;6(264):p. $254 \mathrm{~cm} 12$.

16. Rietschel ET, Bruckner-Tuderman L, Schutte G, Wess G. Translation. Moving medicine forward faster. Sci Transl Med. 2015;7(277):p.277ed2.

17. Morrison RJ, Hollister SJ, Niedner MF, et al. Mitigation of tracheobronchomalacia with 3D-printed personalized medical devices in pediatric patients. Sci Transl Med. 2015;7(285):285ra64. 
18. Michalski MH, Ross JS. The shape of things to come. 3D printing in medicine. JAMA. 2014;312:2213-4.

19. McNeer NA, Anandalingam K, Fields RJ, et al. Nanoparticles that deliver triplex-forming peptide nucleic acid molecules correct F508del CFTR in airway epithelium. Nat Commun. 2015;6:6952.

20. Langer R, Weissleder R. Scientific discovery and the future of medicine. Nanotechnology. JAMA. 2015;313:135-6.

21. D'Ambrosio MV, Bakalar M, Vennuru S, et al. Point-ofcare quantification of blood-borne filarial parasites with a mobile phone microscope. Sci Transl Med. 2015;7(286): 286re4.
22. Weisberg DF. Science in the service of patients: lessons from the past in the moral battle for the future medical education. Yale J Biol Med. 2014;87(1):79-89.

23. Wiencke MC. Teaching the intangibles. Dartmouth Medicine. 2014 (Fall); 32-5.

24. Crawford L. The art of medicine. Medicine at Michigan. Summer 2015. www.medicineatmichigan.org/magazine/2015/summer/artmedicine. Accessed 19 September 2015.

25. Loftus PD, Elder CT, D'Ambrosio T, Langell JT. Addressing challenges of training a new generation of clinician-innovators through an interdisciplinary medical technology design program: bench-tobedside. Clin Transl Med. 2015;4:15. 